\title{
The Role of Innate and Adaptive Immune Response Lymphocytes in Pathogenesis of Hemorrhagic
} Fever with Renal Syndrome

\author{
Mikhail F Ivanov ${ }^{1}$, Irina P Balmasova ${ }^{2 *}$, Alexander V Zhestkov ${ }^{1}$ and Larisa A Rogozina ${ }^{1}$ \\ ${ }^{1}$ Samara State Medical University, Samara, Russia \\ ${ }^{2}$ AIEvdokimov Moscow State University of Medcine and Dentistry, Moscow, Russia \\ ${ }^{3}$ Peoples' Friedship University of Russia, Moscow, Russia \\ *Corresponding Author: Irina P Balmasova, AIEvdokimov Moscow State University of Medicine and Dentistry, Mosfilmovskaya Moscow, \\ Russian Federation.
}

Received: June 20, 2019; Published: July 17, 2019

DOI: $10.31080 /$ ASMS.2019.03.0357

\begin{abstract}
Hemorrhagic fever with renal syndrome (HFRS) is a feral herd infection caused by Hantaviruses. This infection is manifested with hemorrhagic diathesis, hemodynamic abnormalities, and kidney injury. Clinical manifestations of HFRS includes febrile, oliguric, polyuric, and convalescence periods. This is a pattern of immune response at different stages of HFRS. It was demonstrated that the $\mathrm{CD} 3+\mathrm{CD} 8+\mathrm{T}$ cell response was delayed cellular response. This pattern can be accounted for high levels of regulatory $\mathrm{T}$ cells which inhibit both lymphocyte functions and inflammation. This phenomenon should be considered as a protective reaction to reduce systemic damage in HFRS.
\end{abstract}

Keywords: H Emorrhagic Fever with Renal Syndrome; Immune Response; Lymphocyte Phenotypes; Acute Renal Failure

\section{Abbreviations}

ARF: Acute Renal Failure; CD: Cluster Differentiation Marker; CTL: Cytotoxic T Lymphocyte; DAP: Death-Associated Protein; HFRS: H Emorrhagic Fever With Renal Syndrome; IL: interleukin; ILR: Receptor To Interleukin; MIC: MHC Class I-Related Chain; NK: Natural Killer Cell; NKT: Natural Killer T Lymphocyte; NKG2D: Lectin Activating Receptor Expressed; Treg: Regulatory T Cells

\section{Introduction}

Hemorrhagic fever with renal syndrome (HFRS) - Acute feral herd viral disease, manifested by hemorrhagic syndrome, hemodynamic disorders and kidney damage with the development of acute renal failure [7]. The disease is caused by hantaviruses [3]. In Russia, the etiology of HFRS in humans is associated with 5 types of hantaviruses. The most active focal area is located in the Urals and Middle Volga region, which includes the Samara region, where the main causative agent of the disease is type Puumala. It is noted that the disease caused by Hantavirus type Puumala has a relatively mild course, hemorrhagic manifestations are recorded only in $8-9 \%$ of patients, mortality in this form does not exceed $0.5 \%$ [7].

The HFRS pathogens infect mainly the endothelial cells and replicate in them without a cytopathic effect [12]. The endothelium of the capillaries and small vessels is mainly affected; with regard to kidney damage, a very characteristic sign of HFRS is the rupture of intercellular contacts in the renal glomeruli endothelial cells infected with hantaviruses [9]. The number of cells sensitive to these viral pathogens also includes macrophages, dendritic cells, lymphocytes, neutrophils, and platelets [11]. Like many other pathogenic viruses, hantaviruses have an indirect effect on the infected organism through the immune system, which carries out both the innate and adaptive immune response [2,4]. 
In the literature of recent years there are isolated information about the effect of renal noninfectious genesis on the immune status [8], although the significance of acute renal failure in the immunopathogenesis of HFRS has not been practically studied.

The following periods of illness are clinically distinguished : incubation (from 1 to 5 weeks, on average 2-3 weeks), feverish (initial, general toxic ), lasting on average from 3 to 7 days, oliguric (on average 6-12 days ), polyuric (on average, 6-14 days ), recovery period (from 2 months to 2-3 years) [6].

The aim of the work was to analyze the pattern of the immune response in HFRS at different stages of the infectious process and the role of acute renal failure in the observed immunological shifts.

\section{Materials and methods}

Were under surveillance 65 patients with HFRS throughout all stages of the infectious process. The diagnosis of HFRS was verified on the basis of epidemiological data and by determining IgM and IgG antibodies to the hantavirus nucleocapsid protein using paired sera. The comparison group included 9 people with acute renal failure of non-infectious genesis who were admitted to hospital for hemodialysis. The control group of clinically healthy people during laboratory tests consisted of 16 people.

Phenotyping of the immune system cells in the blood of sick and healthy people was carried out using flow cytofluorimetry.

To characterize the adaptive immune response in patients with HFRS at the phenotypic level, the following were examined: the numerical composition of B- (CD19+) and T-lymphocyte (CD3+) populations, T-helper subpopulations $(\mathrm{CD} 3+\mathrm{CD} 4+)$, cytotoxic T- lymphocytes $(\mathrm{CD} 3+\mathrm{CD} 8+)$, regulatory $\mathrm{T}$ cells $(\mathrm{CD} 3+\mathrm{FoxP} 3+)$ with both $\mathrm{CD} 4+$ and $\mathrm{CD} 8+$, as well as expression of the activation marker CD25 by T-lymphocytes (CD3+CD25+) and activating lectin receptor NKG2D (CD314) by cytotoxic T-lymphocytes (CD3+CD8+CD314+). Among the lymphocytes of innate immunity, natural killer T-lymphocytes (EKT, CD3+CD56+), natural killer cells (NK, CD16+CD56+), and NK cells, expressing NKG2D activating lectin receptors (CD16+CD56+CD314+) associated with the induction of the cytotoxic activity of these cells. Statistical analysis of the results was carried out on the basis of the statistical software package SPSS, version 21.

\section{Results and Discussion}

The results of the analysis of the relative content in the blood of adaptive immunity lymphocytes of various populations/subpopulations in patients with HFRS in different periods of the disease in comparison with the control are presented in Figure 1, reflecting the percentage deviations of patients from the control values. Al- most all indicators of basic immunograms of patients with HFRS at a particular stage of the disease are informative and show significant differences with the control, and unique for each category of lymphocytes. Thus, the number of B-lymphocytes was significantly higher than the control values only in the oliguric period, which, according to the pattern of antibody production in HFRS, accounts for the onset of the development of the humoral immune response.

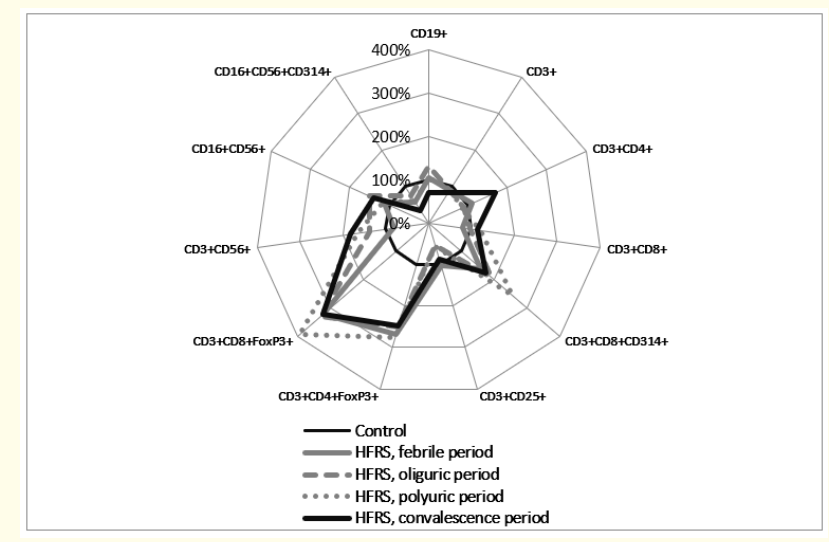

Figure 1: Percentages of deviation from patients with different levels of disease.

The relative number of T-lymphocytes still in the febrile period acquires a tendency to decrease and then remains significantly lower than in the control. This phenomenon may be associated with the migration of T-lymphocytes from the bloodstream to the main target organ for exposure to the HFRS pathogen, the kidney, as well as the possibility of suppressing the lymphocytic response by regulatory T-cells. Both of these causes can also affect the decline in blood of T-helper subpopulations, which become statistically significant in the polyuric period. The number of cytotoxic Tlymphocytes (CTL), on the contrary, significantly increases in the polyuric period.

A certain role in the activation of the cytotoxic subpopulation of T-cells is likely to be played by the expression of this category of lymphocytes activating lectin receptors NKG2D (CD314). The number of CD314+ CTLs significantly increased even during the febrile period and remains at a level of 3.2-4 times the control values during the whole disease.

The most characteristic feature of the adaptive immune response in HFRS is a very significant increase in the presence of regulatory T-lymphocytes (Treg) in the blood with their suppressor activity. Inducible regulatory $\mathrm{T}$ cells in the course of the immune response can be formed by reprogramming both CD4+ T-helpers and CD8+ cytotoxic T-lymphocytes, while maintaining the phenotype of 
these cells. The number of these cells is increased throughout the entire disease, especially since the oliguric period, and the number of CD8+ Treg increases especially significantly. This coincides with a significant drop in the number of activated T-lymphocytes $(\mathrm{CD} 3+\mathrm{CD} 25+)$ in the blood, starting from the oliguric period.

Among lymphocytes of innate immunity deserve special attention natural killer cells (NK) with phenotype CD16+CD56+, whose immunopathogenetic role in HFRS noted in the scientific literature, and natural killer T lymphocytes (NKT) with phenotype CD3+CD56+, the description of the pathogenetic significance of which in this disease was not found in the available sources.

In the oliguric period, NKT tended to increase, although this increase was not significant. In the polyuric period, the increase in the content of these cells in the blood was statistically significant. During the recovery period, the median of NKT share among lym- phocytes was 1.8 times higher than the control values, but because of the pronounced scatter of data, these deviations lost confidence.

The content of NK in the blood significantly increased in the oliguric period, however, the expression of activating NKG2D receptors by these cells decreased. During the polyuric period, the number of NK cells tended to grow, but the expression of activating lectin receptors by these cells continued to fall. Such dynamics persisted in the period of convalescence.

Assessing the results obtained from pathogenetic positions and from the standpoint of planning therapeutic measures, it is important to determine to what extent they are associated with the characteristics of the viral pathogen or the development of acute renal failure. To answer this question, a comparison was made of immunological signs in patients with HFRS in the oliguric period with those in patients with acute renal failure (ARF) of non-infectious genesis (Table 1).

\begin{tabular}{|c|c|c|c|c|}
\hline \multirow{2}{*}{$\begin{array}{l}\text { Lymphocyte } \\
\text { phenotypes }\end{array}$} & \multicolumn{3}{|c|}{ Median [ minimum; maximum] } & \multirow[b]{2}{*}{$\mathbf{p}_{1} \mathbf{p}_{2}$} \\
\hline & $\begin{array}{l}\text { HFRS patients, oliguric } \\
\text { period, } n=65\end{array}$ & $\begin{array}{l}\text { Patiets with nonifectional } \\
\text { renal failure, } n=9\end{array}$ & $\begin{array}{l}\text { Healthy persons (control) } n= \\
16\end{array}$ & \\
\hline \multirow[t]{2}{*}{ CD19+ } & $13.6[5 ; 25]$ & $11.4[7.6 ; 15.2]$ & \multirow[t]{2}{*}{$10.5[2.5 ; 15.7]$} & $0.048^{*}$ \\
\hline & \multicolumn{2}{|c|}{$\mathrm{p}_{3}=0,502$} & & 0.514 \\
\hline \multirow[t]{2}{*}{$\mathrm{CD} 3+$} & $67.8[50 ; 82]$ & $76[30 ; 80.4]$ & \multirow[t]{2}{*}{$75[62 ; 87]$} & $0.033 *$ \\
\hline & \multicolumn{2}{|c|}{$\mathrm{p}_{3}=0,225$} & & 0.867 \\
\hline \multirow[t]{2}{*}{$\mathrm{CD} 3+\mathrm{CD} 4+$} & $36.3[19.5 ; 46.8]$ & $32.8[17.5 ; 52]$ & \multirow[t]{2}{*}{$41[14 ; 57]$} & 0.069 \\
\hline & \multicolumn{2}{|c|}{$\mathrm{p}_{3}=0,874$} & & 0.437 \\
\hline \multirow[t]{2}{*}{$\mathrm{CD} 3+\mathrm{CD} 8+$} & $27[19.6 ; 78]$ & $26.5[12.5 ; 50]$ & \multirow[t]{2}{*}{$28[16 ; 71]$} & 0.678 \\
\hline & \multicolumn{2}{|c|}{$\mathrm{p}_{3}=0.833$} & & 0.956 \\
\hline \multirow[t]{2}{*}{$\mathrm{CD} 3+\mathrm{CD} 8+\mathrm{CD} 314+$} & $24.2[7.2 ; 48.9]$ & $13.5[5.5 ; 49.6]$ & \multirow[t]{2}{*}{$12.6[9.6 ; 27]$} & $<0.001 *$ \\
\hline & \multicolumn{2}{|c|}{$\mathrm{p}_{3}=0.045^{*}$} & & 0,828 \\
\hline \multirow[t]{2}{*}{$\mathrm{CD} 3+\mathrm{CD} 25+$} & $4.1[1.1 ; 8.9]$ & $3[1,4 ; 4.1]$ & \multirow[t]{2}{*}{$7.5[2.6 ; 7.8]$} & $0.042 *$ \\
\hline & \multicolumn{2}{|c|}{$\mathrm{p}_{3}=0.013 *$} & & $0.032 *$ \\
\hline \multirow[t]{2}{*}{$\mathrm{CD} 3+\mathrm{CD} 4+$ FoxP3 + } & $10.7[5.2 ; 16.3]$ & $4.3[3.2 ; 5.4]$ & \multirow[t]{2}{*}{$3.05[2.3 ; 8.1]$} & $<0.001 *$ \\
\hline & \multicolumn{2}{|c|}{$\mathrm{p}_{3}=0.011 *$} & & 0.410 \\
\hline \multirow[t]{2}{*}{ CD3 + CD8 + FoxP3 + } & $14.8[3.5 ; 23.9]$ & $0.6[0.2 ; 4,5]$ & \multirow[t]{2}{*}{$0.45[0.1 ; 4,4]$} & $<0.001 *$ \\
\hline & \multicolumn{2}{|c|}{$\mathrm{p}_{3}=0.011 *$} & & 0.534 \\
\hline \multirow[t]{2}{*}{ CD3 + CD56 + } & $4.7[1.7 ; 30.6]$ & $13[10 ; 27]$ & \multirow[t]{2}{*}{3.4 [2.3; five] } & 0.139 \\
\hline & $\mathrm{p}_{3}=$ & $.002 *$ & & $0.001 *$ \\
\hline \multirow[t]{2}{*}{ CD16 + CD56 + } & $16.9[9 ; 53]$ & $10[3.4 ; 15]$ & \multirow[t]{2}{*}{$12.9[9.5 ; 27.7]$} & $0.045 *$ \\
\hline & $\mathrm{p}_{3}=$ & $.002 *$ & & $0.040 *$ \\
\hline \multirow{2}{*}{$\begin{array}{l}\text { CD16 + CD56 + } \\
\text { CD314 + }\end{array}$} & $7.2[1 ; 16.3]$ & $9.7[4.1 ; 18]$ & \multirow[t]{2}{*}{$9.6[7.7 ; 21.6]$} & 0.079 \\
\hline & \multicolumn{2}{|c|}{$\mathrm{p}_{3}=0,329$} & & 0.780 \\
\hline
\end{tabular}

Table 1: Indicators of the content (\%) in the blood of lymphocytes of the innate and adaptive immune response in patients with HFRS and in the comparison groups.

Note: $\mathrm{n}$ - the number of patients in the group; $\mathrm{p}_{1}$ - probability of differences between HFRS patients and controls; $\mathrm{p}_{2}$ - the probability of differences between ARF patients and controls; $\mathrm{p}_{3}$ - the probability of differences between HFRS and ARF patients; * significance of differences (by Mann-Whitney U-test) at $\mathrm{p}<0.05$. 
The Role of Innate and Adaptive Immune Response Lymphocytes in Pathogenesis of Hemorrhagic Fever with Renal Syndrome

As follows from the table, the impact of acute renal failure of non-infectious genesis on immune processes is manifested primarily in a significant increase in the number of NKT in the blood, which is 38.2 times higher than the content of these cells in blood samples of healthy people and 2.8 times patients with HFRS. The tendency to increase in the number of these cells was previously noted by us and in HFRS, but it was not reliable and it can now be fully associated with the occurrence of acute renal failure in this disease.

All other immunological signs of the HLPS oliguric period are an increase in the relative number of B-lymphocytes with a drop in the number of T-cells, an increase in CTL expression of activating lectin receptors NKG2D (CD314) and a decrease in the expression of IL-2 receptors in T-cells (CD25), high blood levels of regulatory T-cells (FoxP3+) and NK cells - can be interpreted as manifestations of hantavirus infection.

For a more accurate interpretation of the data obtained from the point of view of the role of the functional state of the lymphocytes of the innate and adaptive immune response in the pathogenesis of HFRS, a correlation analysis was conducted using the Spearman correlation coefficient ( $r$ ), the results of which are presented in Figure 2.

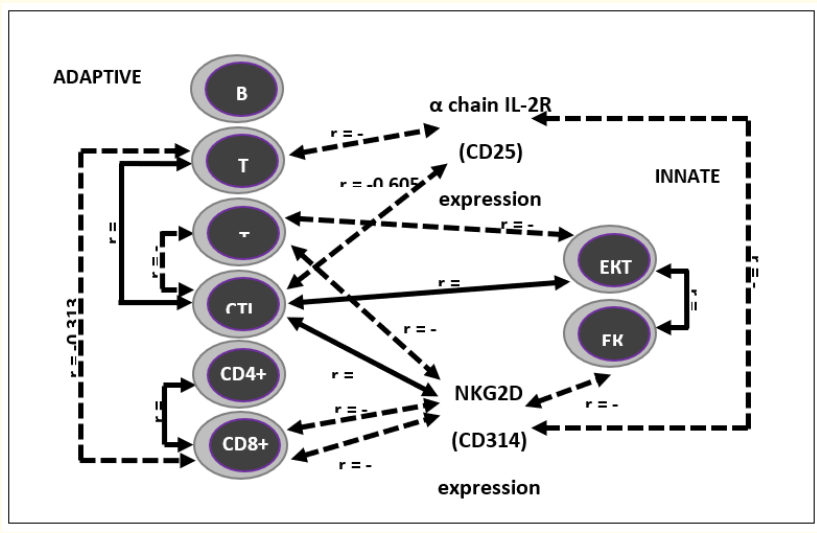

Figure 2: Significant correlation between the receptors in the HFRS: $r$ - Spearman correlation coefficient, positive correlation, negative correlation).
Despite the conventionality of the values of correlations, their analysis provides quite a lot of information about the relationship of lymphocytes of different phenotypes in HFRS. Of particular interest were the relationship of expression of activating receptors of different nature with the content of different populations/subpopulations of lymphocytes in the blood in HFRS and, above all, lymphocytes with cytotoxic activity.

The presence of an activating receptor CD25 ( $\alpha$-chain receptor for IL-2) was determined on the T-lymphocyte membrane $(C D 3+C D 25+)$ and showed a decrease in the number of cells expressing this receptor (negative correlation, $r=-0,328$ ). Judging by the analysis of correlations, the fall in CD25 expression was due to a subpopulation of cytotoxic T-lymphocytes $(r=-0.625)$.

Expression of activating receptors CD314 ( NKG2D) was determined on the membrane of cytotoxic T-lymphocytes and natural killer cells, in the first case a positive correlation was established $(r=0.720)$, and in the second - negative ( $r=-0.447)$, which fully corresponds to the dynamics of these cells in the blood. The expression of NKG2D receptors was in a negative relationship with a high content of regulatory $\mathrm{T}$ cells in the blood, which, according to correlations, exerted an inhibitory effect on T lymphocytes as a whole. The balance between the expression of NKG2D and CD25 (r $=-0.558)$ is also interesting.

Should be noted such a phenomenon as a positive correlation of ECT associated with the development of renal failure, and cytotoxic T-lymphocytes $(\mathrm{r}=0.376)$.

Discussing the results, we should focus on three key points. The first aspect concerns the role of cells with cytotoxic activity - cytotoxic T-lymphocytes (CTL) and natural killer cells (NK) in the immunopathogenesis of HFRS. CTLs belong to the adaptive immune response cells and are induced by viruses, presented by the affected cells, and NKs are innate immunity lymphocytes, the cytotoxic effects of which are triggered by a special system of inhibitory and activating receptors that respond to signals from histocompatibility class I stress-induced molecules, patterns of cellular damage [1]. The percentage of CTL in the blood did not significantly change with HFRS or non-infectious genesis of ARF against the background of the decline in their expression of the early activation marker CD25 by other T lymphocytes, but the expression of the activating

Citation: Irina P Balmasova., et al. "The Role of Innate and Adaptive Immune Response Lymphocytes in Pathogenesis of Hemorrhagic Fever with Renal Syndrome". Acta Scientific Medical Sciences 3.8 (2019): 120-125. 
lectin receptor NKG2D by cytotoxic T lymphocytes significantly increased. The percentage content of the NK cells on the contrary, increased when HFRS against decrease NKGD2 expression.

The fact is that many cells of the immune system can demonstrate the ability to express the lectin receptor NKG2D. First of all, cells with cytotoxic activity belong to them - NK, NKT, $\gamma \delta$ T cells, CD8+ T cells, certain categories of CD4+ T cells [5,13], however, there are differences in the functioning of NKG2D on the membrane of these cells. In particular, this receptor realizes the signals coming from it in natural killer cells and cytotoxic T-lymphocytes in different ways. Ligands for this receptor are, as a rule, stress-inducible MICA and MICB molecules on the surface of virus-infected and other abnormal cells [1]. In case of viral infections, the NK cells includes 2 signaling pathways - with the participation of adapter molecules Dap 12 (via Syk) and Dap 10 (through PI3-kinase), and in CTL - only with the participation of Dap 10 [10]. As a result, in NK cells, the interaction of NKG2D with its ligands leads both to a cytotoxic effect and to the release of cytokines and cytotoxic T cells use signals coming through NKG2D only for their activation [14].

According to our data, the expression of NKG2D by cells of the adaptive immune response - CTL (CD3+CD8+CD314+) increases dramatically in HFRS mainly in response to the infection of endothelium cells with hantavirus, since in non-infectious genesis of ARF this growth is may be triggered by own damaged cells with the participation of other factors, expressed to a much lesser extent. This hypothesis is confirmed by the absence or even a decrease in the proportion of NK cells expressing this receptor (CD16+CD56+CD314+). Given the current understanding of the dual (virus-eliminating and damaging) role of CTL in HFRS [6], it can be assumed that the balance in the activation of CTL/NK is aimed at reducing the immuno-damaging effects.

From this point of view, an increase in the number of regulatory T cells (CD3+FoxP3+) in HFRS in both CD 4+ and, especially, CD8+ can be interpreted. With the participation of these cells, which suppress, including, cytotoxic reactions, the immune system limits the effects of damage to the endothelium and the renal glomeruli [8]. It can be assumed that induction of regulatory T-cells is caused by hantavirus, as it is observed in HFRS, but not in non-infectious genesis of ARF.
Another interesting point concerns NKT (CD3+CD56+) lymphocytes, capable of producing both IFN $\gamma$, a cytokine of type 1, inducing cellular immune responses, and type 2 cytokines (IL-10, IL-4), which influence the development of the humoral immune response [1]. In the experiment, it was shown that type 2 NKTs manifest themselves most effectively in acute renal failure, thereby suppressing hypoxia-induced death of epithelial cells of the renal tubules [15]. Judging by our data, this mechanism, the possibility of which is confirmed by a very significant increase in the number of NKTs for non-infectious respiratory infections, is significantly reduced in HFRS, and protective reactions aimed at limiting cellular damage and providing, in fact, the patient's survival above the mechanisms.

\section{Conclusion}

Immunological changes in hemorrhagic fever with renal syndrome, on the one hand, due to hantavirus and include the drop in the number of T-lymphocytes due to a subpopulation of $\mathrm{T}$ helper cells (CD3+CD4+), an additional stimulation of the expression activating lectin receptor NKG2D by cytotoxic T cells but not natural killers. On the other hand, changes in the immune status can also be associated with kidney damage, manifested by acute renal failure and associated with an increase in the number of NKT (CD3+CD56+). A very important feature of the immune response in HFRS is a significant and sustained growth, starting from a febrile period, the content of regulatory $\mathrm{T}$ cells in the blood $(\mathrm{CD} 3+\mathrm{CD} 4+\mathrm{FoxP} 3+$ and CD3+CD8+FoxP3+). This and other features of the immune response show their possible focus on limiting the immune mechanisms of systemic cell damage in HFRS.

\section{Conflict of Interest}

The authors declare no conflict of interest

\section{Bibliography}

1. Malova ES., et al. "Association between expression of lectin type receptors by natural killers and intensity of liver fibrosis during chronic hepatitis C". Zh Mikrobiol Epidemiol Immunobiol 6 (2010): 48-52.

2. Ivanov MF, et al. "Phenotype facilities of lymphocytes in hemorrhagic fever with renal syndrome". Georgian medical news 266 (2017): 104-110. 
3. Clement J., et al. "Acute hantavirus infection presenting as haemolytic-uraemic syndrome (HUS): the importance of early clinical diagnosis". European journal of clinical microbiology and infectious diseases 37.1 (2018): 135-140.

4. Easterbrook JD., et al. "The Regulatory T cells Enhance the persistence of zoonotic pathogen Seoul virus in ITS reservoir host". Proceedings of the National Academy of Sciences of the United States of America 104.39 (2007): 15502-15507.

5. Jamieson AM., et al. "The role of the NKG2D immunoreceptor in immune cell activation and natural killing". Immunity 17.1 (2002): 19-29.

6. Jiang $\mathrm{H}_{\text {., }}$ et al. "Hemorrhagic with fever renal syndrome: pathogenesis and clinical picture". Frontiers in cellular and infection microbiology 6 (2016): 1-9.

7. Kariwa H, et al. "Epidemiological study of the hantavirus infection in the Samara Region of European Russia". The Journal of veterinary medical science 71.12 (2009): 1569-1578.

8. Kinsey GR., et al. "Expanding role of T cells acute kidney injury". Current opinion in nephrology and hypertension 23.1 (2014): 9-16.

9. Krautkrandmer E., et al. "Pathogenic old world hantaviruses infect renal glomerular and tubular cells and induce disassembling of cell-to-cell contacts". Journal of virology 85.19 (2011): 9811-9823.

10. Lanier L. "NK cell recognition". Annual review of immunology 23 (2005): 225-274.

11. Manigold T., et al. "Human hantavirus infections: epidemiology, clinical features, pathogenesis and immunology". Swiss medical weekly144 (2014): 13937-13955.

12. Pensiero MN., et al. "Hantaan virus infection of human endothelial cells". Journal of virology 66.10 (1992): 5929-5936.

13. Raulet DH. "Roles of the NKG2D immunoreceptor and its ligands". Nature reviews Immunology 3.10 (2003): 781-790.

14. Sentman CI., et al. "NKG2D CARs as cell therapy for cancer ". The cancer journal 20.2 (2014): 156-159.

15. Yang H., et al. "Sulfatide -reactive natural killer T cells abrogate ischemia-reperfusion injury". Journal of the American Society of Nephrology 22.7 (2011): 1305-1314.

\section{Volume 3 Issue 8 August 2019}

(C) All rights are reserved by Irina $P$ Balmasova., et al. 